\title{
2-CONNECTED HAMILTONIAN CLAW-FREE GRAPHS INVOLVING DEGREE SUM OF ADJACENT VERTICES
}

\author{
TAO TIAN \\ School of Mathematics and Statistics \\ Beijing Institute of Technology \\ Beijing 102488, P.R. China \\ Faculty of EEMCS, University of Twente \\ P.O. Box 217, 7500 AE Enschede, The Netherlands \\ e-mail: taotian0118@163.com \\ AND \\ LIMING XIONG \\ School of Mathematics and Statistics \\ Beijing Key Laboratory on MCAACI \\ Beijing Institute of Technology \\ Beijing 102488, P.R. China \\ e-mail: lmxiong@bit.edu.cn
}

\begin{abstract}
For a graph $H$, define $\bar{\sigma}_{2}(H)=\min \{d(u)+d(v) \mid u v \in E(H)\}$. Let $H$ be a 2-connected claw-free simple graph of order $n$ with $\delta(H) \geq 3$. In [J. Graph Theory 86 (2017) 193-212], Chen proved that if $\bar{\sigma}_{2}(H) \geq \frac{n}{2}-1$ and $n$ is sufficiently large, then $H$ is Hamiltonian with two families of exceptions. In this paper, we refine the result. We focus on the condition $\bar{\sigma}_{2}(H) \geq \frac{2 n}{5}-1$, and characterize non-Hamiltonian 2-connected claw-free graphs $H$ of order $n$ sufficiently large with $\bar{\sigma}_{2}(H) \geq \frac{2 n}{5}-1$. As byproducts, we prove that there are exactly six graphs in the family of 2-edge-connected triangle-free graphs of order at most seven that have no spanning closed trail and give an improvement of a result of Veldman in [Discrete Math. 124 (1994) 229-239].
\end{abstract}

Keywords: Hamiltonian cycle, degree sum, dominating closed trail, closure.

2010 Mathematics Subject Classification: 05C45. 


\section{REFERENCES}

[1] J.A. Bondy and U.S.R. Murty, Graph Theory (Springer, 2008).

[2] P.A. Catlin, A reduction method to find spanning Eulerian subgraphs, J. Graph Theory 12 (1988) 29-45. doi:10.1016/S0012-365X(95)00149-Q

[3] P.A. Catlin, Z. Han and H.-J Lai, Graphs without spanning Eulerian trails, Discrete Math. 160 (1996) 81-91. doi: $10.1002 /$ jgt. 22120

[4] Z.-H. Chen, Hamiltonicity and degrees of adjacent vertices in claw-free graphs, J. Graph Theory 86 (2017) 193-212. doi:10.1002/jgt.22120

[5] G.H. Fan, New sufficient conditions for cycles in graphs, J. Combin. Theory Ser. B 37 (1981) 221-227. doi:10.1016/0095-8956(84)90054-6

[6] R. Faudree, E. Flandrin and Z. Ryjáček, Claw-free graphs - A survey, Discrete Math. 164 (1997) 87-147. doi:10.1016/S0012-365X(96)00045-3

[7] O. Favaron, E. Flandrin, H. Li and Z. Ryjáček, Clique covering and degree conditions for Hamiltonicity in claw-free graphs, Discrete Math. 236 (2001) 65-80. doi:10.1016/S0012-365X(00)00432-5

[8] R. Gould, Recent advances on the Hamiltonian problem: Survey III, Graphs Combin. 30 (2014) $1-46$. doi:10.1007/s00373-013-1377-x

[9] F. Harary and C.St.J.A. Nash-Williams, On Eulerian and Hamiltonian graphs and line graphs, Canad. Math. Bull. 8 (1965) 701-710. doi:10.4153/CMB-1965-051-3

[10] O. Kováŕík, M. Mulač and Z. Ryjáček, A note on degree conditions for Hamiltonicity in 2-connected claw-free graphs, Discrete Math. 244 (2002) 253-268. doi:10.1016/S0012-365X(01)00088-7

[11] H. Li, M. Lu, F. Tian and B. Wei, Hamiltonian cycles in 2-connected claw-free graphs with at most $5 \delta$ - 5 vertices, Congr. Numer. 122 (1996) 184-202.

[12] M.M. Matthews and D.P. Sumner, Longest path and cycle in $K_{1,3}$-free graphs, J. Graph Theory 9 (1985) 269-277. doi:10.1002/jgt.3190090208

[13] O. Ore, Note on Hamilton circuits, Amer. Math. Monthly 67 (1960) 55. doi: $10.2307 / 2308928$

[14] Z. Ryjáček, On a closure concept in claw-free graphs, J. Combin. Theory Ser. B 70 (1997) 217-224. doi:10.1006/jctb.1996.1732 
[15] Y. Shao, Claw-free graphs and line graphs, Ph.D dissertation (West Virginia University, 2005).

[16] H.J. Veldman, On dominating and spanning circuits in graphs, Discrete Math. 124 (1994) 229-239. doi:10.1016/0012-365X(92)00063-W

[17] C.Q. Zhang, Hamilton cycles in claw-free graphs, J. Graph Theory 12 (1988) 209-216.

doi:10.1002/jgt.3190120211

Received 19 May 2017

Revised 6 February 2018

Accepted 6 February 2018 